\title{
On the successive perception of simultaneous stimuli '
}

HARRIS B. SAVIN

UNIVERSITY OF PENNSYLVANIA

When Ss hear two messages concurrently over different "channels" (e.g., different ears, different voices, etc.), they tend to report first one message and then the other, rather than collating the two. This tendency has nothing to do with the nature of the differences between the two channels. It is a manifestation of a tendency for the auditory system to group successive, rather than simultaneous inputs. The same sequential grouping is found in the $S s^{\prime}$ order of report when there is no stimulus property to differentiate between two channels.

When Ss listen to rapidly presented sequences of dichotic digits and then recall them, they tend strongly to recall first all the digits heard through one ear and then all the ones heard through the other ear. Moreover, when they are required to recall first the first pair, then the next, and so on (i.e., to collate the messages heard at the two ears), they are highly inaccurate (Broadbent, 1956). Hereafter, this preferred order of recall will be called "channel-bychannel"-each ear being a channel.

Essentially similar results have been reported in other experiments in which Ss heard two simultaneous sequences and the channel-defining characteristic was not the difference between right and left ears but a difference in the talker's voice quality (produced by filtering low frequencies on one channel; Broadbent, 1956), or where one channel consists of words and the other, digits (Gray \& Wedderburn, 1960; Yntema \& Trask, 1963). That is to say, if a $S$ hears a sequence of pairs, each consisting of two digits spoken in different voices (or of a digit and a word), he tends to recall first all the digits spoken in one voice and then all the ones spoken in the other voice (respectively, all the words and then all the digits or vice versa). When allowed to recall in any order he wishes, he almost never collates two concurrent sequences; when he is required to collate them, he is inaccurate.

Most theorists have assumed that the various channeldefining characteristics are crucial in an explanation of these findings. Broadbent, for example, belleves that there is a separate immediate memory at each ear and that it takes about $1 / 2 \mathrm{sec}$ to switch attention from one ear to the other. Hence, the $S$ finds it most efficlent to first percelve all the digits coming into one ear and then turn to some relatively unprocessed memory-representation of the digits in the other ear's Immediate memory, perceiving those digits only then. The order of recall, then, is the order of perception. Broadbent believes, therefore, that the explanation of channel-by-channel recall is quite different when the channel-defining characteristic is right ear/left ear than when it is, say words/digits. (See Broadbent \& Gregory, 1964, for his explanation of the latter, which also depends crucially upon the precise characteristic that defines the channels.) Yntema and Trask (1963) on the other hand propose a single explanation of all the cases in which channelby-channel recall is easiest. They assume, essentially, that the various items are stored in immediate memory together with "tags" indicating such things as what ear they came through, what volce they were spoken in, and so on. They further assume that, in general, Ss have some difficulty in keeping track of the items that they have already recalled. Channel-by-channel retrieval reduces this difficulty, they claim, by allowing the $S$ to remember (for example) that he has now recalled all the right-ear digits instead of requiring him to remember precisely which digits he has recalled. Again, the channel-defining characteristics are crucial. Yntema and Trask predict that channelby-channel recall will be the most efficient order for just those channel-defining characteristics that are represented by "tags" in immediate memory.

The thesis of the present paper is that channeldefining characteristics-and, indeed, channels-are largely, if not wholly, irrelevant to the preferred order of recall. The preferred order of recall, it will be shown, is a reflection of a principle of auditory perceptual organization; the auditory system tends to group together stimuli that occurred sequentially in time, rather than stimuli that occurred simultaneously. (Note that such a principle is desirable in a speech-perception system; words that occur simultaneously must, outside the laboratory, have come from different mouths and hence must be unrelated.) The only role played by the channels in the experiments discussed above, on this view, is to resolve an ambiguity that is allowed by this principle of grouping. That is to say, channels (of practically any sort) will determine which digit of the flrst pair is grouped with which one of the second pair, and so on. Even in the absence of any sort of channeldefining characteristic, however, one digit of the first pair will be grouped with one digit of the second. Elimination of all channel-defining characteristics will simply make it a matter of chance which one of the second pair is grouped with which one of the first. 


\section{Procedure}

Each trial consisted of two pairs of simultaneous digits. The second pair began $1 / 2$ sec after the beginning of the first. One digit of each simultaneous pair was recorded on each track of a stereo tape recorder (Sony Model TC-200), and both tracks were played back through a single loudspeaker. The same digit never occurred twice on a single trial. All four digits were recorded by the same talker. In order to preclude the possibility that some kind of articulatory continuity might link the two successive digits on the same track (and hence define a channel), the tape recorder was stopped for several seconds after each digit was recorded, and then restarted. This is not as difficult as it sounds, provided the recorder, like the Sony, is equipped with a very fast clutch mechanism. The talker quickly learns to engage the clutch just as he says a digit. All four digits of a trial, therefore, were spoken on independent occasions. That the digits on the two tracks were properly synchronized was verified by watching two adjacent VU meters, one of which measured the signal on each track of the tape.

The Ss were 15 undergraduates at the University of Pennsylvania. Ss were told to repeat all four digits of each trial as quickly as possible after they were presented and in whatever order they found easiest. No latencies were recorded but, for reasons to be explained below, the $E$ rather ostentatiously busied herself with a stopwatch throughout the experiment. Ss were told nothing about the physical structure of a trial, except that it consisted of four digits "all within a short time," and they were told explicitly to recall in whatever order they found easiest.

There were 41 trials in all. The first six were practice trials, the results of which were discarded. Each practice trial was played back to the $S$ repeatedly until he was able to repeat all four digits correctly. (Practice was necessary because, in the absence of any localization cue, the first few trials that one hears are almost unintelligible.) The 35 trials of the experiment proper followed immediately after the practice trials. Each of these was presented only once to each S. Ss were not told whether their responses were correct or not.

\section{Results}

Preferred order of recall. There were a total of 525 experimental trials. All four digits were correctly recalled on 319 of them. On 217 of these, the Ss grouped together successive pairs (i.e., they recalled first one digit of the first simultaneous pair followed by one digit of the second, and then the other digit of the first pair, followed by the other digit of the second). That is to say, 217 of the correct trials had the digits in the order that, had had there 1 sen any channels, would be called "channel-by-channel." On 58 trials, simultaneous pairs were grouped to- gether. The remaining 44 correct trials appear to be instances of grouping successive digits together, but they are less unequivocal. The first two digits were from successive pairs, as were the last two, but one pair of response-digits was in reverse order. For example, if the stimulus had consisted of (simultaneous) 4 and 8 , followed by (simultaneous) 3 and 9 , the order of recall might be 4938 .

Ignoring these 44 equivocal trials, $79 \%$ of the ones that can be classified unequivocally are instances of successive grouping; only $21 \%$ group together simultaneous pairs. There are, however, individual differences. Nine of the 15 Ss grouped successively at least $90 \%$ of the time. For the other six Ss, these percentages were, respectively, $67,62,36,29$, and 0 . The Ss who most often reported all four digits accurately tended strongly to be the ones who grouped successive pairs. The rank-order correlation between the ratio of sequential to simultaneous grouping and the total number of correct trials was $0.63(p<.02$, twotailed). The five worst Ss, in terms of overall performance, as a group produced 76 trials on which all four digits were reported, of these, 30 grouped simultaneous digits together, 26 grouped successive digits, and 20 were equivocal in the sense mentioned above. That is to say, these Ss produced fewer than a fourth of the total number of correct trials, but more than half of the trials in which digits were grouped in simultaneous pairs.

There is reason to suspect that the Ss who usually grouped simultaneous pairs may, in fact, have perceived successive groups and then rearranged the digits before recalling. The fact that they made more errors than did the Ss who reported successive groups itself suggests this. Better evidence comes from preliminary experiments just like this one except that Ss wrote their responses instead of saying them and they were not told to respond quickly. Several of these Ss, although they wrote their responses in the sequential-grouping order, did not write from left to right. Rather, they skipped about on the page so that, the order in which they wrote the digits notwithstanding, the digits appeared on the page grouped in simultaneous pairs. They wrote 1234, in other words, by writing first 1 , then 3 well to the right of 1 , then 2 between the 1 and the 3 , and finally 4 to the right of 3. Other Ss illustrated the same point by writing their digits from left to right, in successive groups, and then drawing arrows to indicate a permutation into simultaneous groups. Both of these kinds of Ss evidently found it easier to recall in successive groups but, for some reason, preferred that their written records show simultaneous groups. No $S$ ever made the opposite rearrangement.

This seemingly perverse behavior is quite explicable in the light of the phenomenal experience of listening to these stimuli. The author and all the articulate Ss seem to agree about what they sound like. One $S$ 
aptly described it as analogous to listening to the melody and harmony of a piece of music. ${ }^{2}$ One does not hear first the melody and then the harmony. Rather one hears both concurrently but groups all the notes of the melody as one perceptual entity and all the notes of the harmony as another. Likewise in the present experiment, one does not perceive the two successive groups successively. Rather, one hears them concurrently. One does hear both members of the first simultaneous pair before either member of the second (although one seldom, if ever, perceives both members of a simultaneous pair simultaneously). Temporal order of perception is simply not related straightforwardly to the principles of perceptual grouping. Nonetheless - and here the musical analogy failsone knows that the successive grouping is arbitrary and imposed by oneself. Hence, it may seem perverse to let it govern one's order of report. The "reasonable" order of report is what is believed to have been the physical order of presentation, not the perceptually natural one. It was precisely to discourage this kind of "reasonableness" that the Ss in the present experiment were told to respond as quickly as possible and to say their responses instead of writing them. But there is no very satisfactory way of knowing whether this procedure completely eliminated postperceptual rearrangements. The straightforward procedure of asking the Ss at the end of the experiment what they had been doing proved surprisingly uninformative. Many of them are simply unable to talk intelligibly about it until they have been given such an elaborate explanation of what is meant by perceptual group that their introspective reports could not possibly be considered unblased.

On repetition of digits within a trial. Yntema and Trask (1963), it will be remembered, proposed that channel-by-channel recall was the optimal strategy because it spared the $S$ the need to remember which digit of a simultaneous pair he had already recalled. Instead, he could simply scan through his immediate memory twice, first retrieving all the items "tagged" with one channel's identifying mark and a second time retrieving all the items of the other channel. Their theory, at least in its simplest form, would predict that, in the present experiment, where no channeldifferentiating tags could have been stored, Ss should often erroneously recall the same digit twice on a single trial. Only one such error was observed in the 525 trials of this experiment.

Might there really be channels in the stimulus? It is not quite clear whether the natural grouping is always or only usually of successive, rather than simultaneous pairs. Whatever view one has of various doubtful points, however, there seems to be no doubt that successive grouping is preferred. It might be argued, however, that, in spite of all precautions, the stimuli were defective-that there were, in fact, channels.
The likeliest source of such artifactual channels is in the tape recorder. Both digits of a simultaneous pair were played through the same loudspeaker but through different playback heads and amplifiers. If channels were introduced here, there should be a tendency, when Ss recall successive groups, for them to group together digits that were on the same track of the tape recorder. If there is no relevant difference between the two playback circuits, a digit on Track 1 of the tape recorder should be just as likely to be grouped with the following digit on Track 2 as with the following digit on Track 1. In fact, within the 217 trials on which all four digits were recalled in successive groups, the probability of grouping the digit of the first pair that was on Track 1 with the digit of the second pair that was on the same track (rather than with the digit of the second pair on the other track) was 0.51 . There is, then, no such bias.

The evidence of the preceding paragraph shows that artifactual channels, if they exist, are unrelated to the circuitry of the tape recorder. It still might be thought, however, that on at least some of the trials, some unknown flaw in the stimulus defines a channel. If this were true, then the data would indicate that the sequential grouping was not in fact ambiguous-that, for each trial separately, all of the Ss who grouped the digits sequentially tended to group each member of the first simultaneous pair with the same member of the second simultaneous pair. For example, if the first simultaneous pair had been 1 and 2 and the second had been 3 and 4 , the Ss who grouped sequentially, on the most extreme version of this hypothesis, either would all group 1 with 3 and 2 with 4 (recalling 1324 or 2413) or else would all group 1 with 4 and 2 with 3 (recalling 1423 or 2314). It is impossible to show convincingly that none of the trials are, in this sense, biased. Casual inspection of the data reveals no obviously biased trials, but the sample size for each trial is so small that failure to reject such null hypotheses would not be very persuasive evidence that those null hypotheses were true. A more satisfactory test of the assumption that sequential grouping occurs only when there are artifactual stimulus-channels can be made in the following way: Let $a_{i}$ be the extent to which different Ss who group the digits of the ith trial sequentially group them the same way (the ratio of the more popular grouping-pattern to the less popular) and let $b_{i}$ be the ratio of successive to simultaneous grouping on the ith trial. If the observed tendency to group sequentially is due to the presence of some artifactual channels, then a and b should be positively correlated. The observed correlation is $-0.08(.07>p>.06$, two-tailed). Even if they exist on some trials, therefore, artifactual stimulus-channels do not explain the observed tendency 
to group sequentially. The two theoretically possible secuential organizations of a trial may not always be equally likely, but sequential grouping occurs just as often on the trials where both are equally likely as on the trials (If any) where some unknown stimulus-property favors one organization over the other.

\section{Conclusion}

The often-demonstrated tendency for Ss to recall concurrent messages successively, rather than collating them, does not depend on the existence of any stimulus-property that defines two channels, one for each message. In the present experiment, the same response-tendency is observed, but there is no such stimulus-property. Even in the absence of channeldefining stimulus-properties, the auditory system tends to group successive, rather than simultaneous, inputs.

\section{References}

Broadient, D. E. Successive responses to simulteneous stimuli. Qutut. J. exp. Psychol., 1956, 8, 145-152.

Broadbent, D. E., \& Gregory, M. Stimulus set and response set: the alternation of attention. Quart. J. exp. Psychol., 1964, 16, 309-317.

Gray, J. A.. \& Wedderburn, A. A. I. Grouping strategies with simultaneous stimuli. Quart, J. exp. Psychol., 1960, 12, 180-184. Yntema, D. B., \& Trask, F. P. Recall as a search process. $J$. verbal Learn. verbal Behav., 1963, 2, 65-74.

\section{Notes}

1. This research was supported by National Science Foundation grant NSF Gs-857 to the University of Pennsylvania. The author is indebted to Jane Carol Catlin for her assistance in all phases of this work.

2. This observation is more insightful than the subject seems to have realized. Polyphonic music would evidently be impossible but for the principle of perceptual grouping that is the topic of this paper.

(Accepted for publication June 26, 1967.) 